\title{
Fibroblasts Expressing PDGF-Receptor-Alpha Diminish During Alveolar Septal Thinning in Mice
}

\author{
STEPHEN E. MCGOWAN AND DIANN M. MCCOY \\ Department of Veterans Affairs Research Service and Department of Internal Medicine, University of Iowa Carver College of Medicine, \\ Iowa City, Iowa 52242
}

\begin{abstract}
In mice, secondary alveolar septal formation primarily occurs during a brief postnatal period and is accompanied by transient expansion of the interstitial lung fibroblast (LF) population. PDGF-A, which solely signals through PDGF-receptor-alpha (PDGF-R $\alpha$ ), is required for expansion, but the receptor's relevant downstream targets remain incompletely defined. We have evaluated the proliferation, apoptosis, and differential response to the selective protein tyrosine kinase inhibitor, imatinib, by $p d g f r \alpha$-expressing LF ( $p d g f r \alpha-\mathrm{LF}$ ) and compared them with their nonexpressing LF counterparts. Our objective was to determine whether diminished signaling through PDGF-R $\alpha$-mediated pathways regulates the decline in myofibroblasts, which accompanies septal thinning and ensures more efficient alveolar gas exchange. Using quantitative stereology and flow cytometry at postnatal d 12 and 14, we observed that imatinib caused a selective suppression of proliferation and an increase in apoptosis. The number of the alpha smooth muscle actin ( $\alpha \mathrm{SMA})$ producing $p d g f r \alpha-\mathrm{LF}$ was also reduced. Using cultures of neonatal mouse LF, we showed that imatinib did not suppress PDGF-R $\alpha$ gene expression but reduced PDGF-A-mediated Akt phosphorylation, potentially explaining the increase in apoptosis. Our findings are relevant to bronchopulmonary dysplasia in which positive pressure ventilation interferes with myofibroblast depletion, septal thinning, and capillary maturation. (Pediatr Res 70: 44-49, 2011)
\end{abstract}

$\mathrm{M}$ ammalian secondary pulmonary alveolar septa form from existing saccules through eruption (lifting off from the primary septum), elongation, and thinning, thereby increasing the gas exchange surface (1). This requires coordinated expansion of the epithelial and endothelial surfaces as well as a supporting structural core. The structural core includes interstitial fibroblasts, which contain stabilizing cytoskeletal elements and secrete extracellular matrix structural proteins. Distinct populations of interstitial lung fibroblasts (LF) have been characterized based on Thy-1 (CD90) (2), the abundance of intracellular neutral lipid droplets, (3) or the presence of alpha-smooth muscle actin ( $\alpha$ SMA, ACTA2) (4). All these phenotypic variants involve differences in PDGF-R $\alpha$ signaling, which is critical for secondary alveolar septation $(2,5)$.

PDGF-A solely signals through PDGF-R $\alpha$, suggesting that PDGF-R $\alpha$ regulates one or more required interstitial fibroblast

Received September 7, 2010; accepted January 31, 2011.

Correspondence: Stephen McGowan, M.D., Division of Pulmonary, Critical Care, and Occupational Medicine, C33B GH, Department of Internal Medicine, University of Iowa Hospitals and Clinics, 200 Hawkins Dr., Iowa City, IA 52242; e-mail: stephenmcgowan@uiowa.edu

Supported by a Merit Review Award from the Department of Veterans Affairs and a Carver Foundation Collaborative Award from the University of Iowa Carver College of Medicine. processes, for example proliferation, migration, extracellular matrix production, or cell loss. Because lung $p d g f-r \alpha$ mRNA declines during P1 through P14 (5), whereas PDGF-AA mRNA remains constant (6), the decline in $p d g f-r \alpha$ expressing LF ( $p d g$ fr $\alpha$-LF) does not result from diminished PDGF-AA ligand. However, the abundance of $p d g f r \alpha-\mathrm{LF}$ is influenced by receptor activity. When PDGF-R $\alpha$ is constitutively activated, the $p d g f r \alpha$-LF subpopulation and septal thickness both increase (7). This suggests that there is a link between PDGF-R $\alpha$ receptor signaling and the abundance of LF bearing the receptor. To explore the mechanism(s) behind this link, we reasoned that if PDGF-R $\alpha$ kinase activity regulates the abundance of PDGF-R $\alpha$ LF, then selectively manipulating protein tyrosine kinase activity should preferentially alter the abundance of LF expressing the receptor. We have used the protein tyrosine kinase inhibitor, imatinib, to study the function of $p d g f r \alpha$-LF at the beginning of alveolar thinning, P12-P14.

We have examined the diminution in PDGF-R $\alpha$-LF, which accompanies septal thinning. Others showed that unbridled signaling by PDGF-A through PDGF-R $\alpha$ results in excessive thickening of the septal core, which leads to late gestational or early postnatal death from respiratory failure $(7,8)$. We hypothesized that PDGF-R $\alpha$ signaling must be dampened to achieve the optimal septal thickness for gas exchange. We further hypothesized that $p d g f-r \alpha$ expressing myofibroblasts diminish through decreased proliferation and increased apoptosis. Although we took an indirect approach, it enabled septal formation to be studied in its entirety, which is not possible when PDGF-A signaling is either completely negated (5) or constitutively activated (7).

Although septal thinning is essential, it is unclear how it is coordinated with formation of a mature capillary bed (9). Some cellular features of the thinning process have been described. There is preferential apoptosis of the lipid interstitial fibroblast subpopulation (3). Septal thinning is accompanied by remodeling of the capillary network with a reduction in septal interstitial extracellular matrix proteins and closer

Abbreviations: Abl, Abelson kinase; Arg, Abelson-related gene; $\boldsymbol{\alpha S M A}$, ACTA2, alpha-smooth muscle actin; BPD, bronchopulmonary dysplasia; DAPI, 4',6-diamidino-2-phenylindole, diacetate; FACS, fluorescence activated cell sorting; GFP, green fluorescent protein; LSCM, laser scanning confocal microscope; LF, lung fibroblast; NF-кB, nuclear factor kappa-lightchain-enhancer of activated B cells; PDGF-R $\boldsymbol{\alpha}$, PDGF-receptor-alpha; $p d g f r \alpha$-LF, $p d g f-r \alpha$ expressing LF; PARP, poly-ADP ribose polymerase; P12, postnatal d 12 
apposition between capillaries and the type-1 epithelium (9). Septal thickening that accompanied overexpression of connective tissue growth factor during P1-P14 seemed to inhibit alveolar capillary formation (10). Understanding the thinning process is important because thickened walls are observed in mechanically ventilated lungs of children (11) and baboons (12) with bronchopulmonary dysplasia (BPD).

\section{MATERIALS AND METHODS}

Reagents. Imatinib and oridonin were obtained from LC Laboratories (Woburn, MA) and Calbiochem (La Jolla, CA), respectively; other reagents were described (13). The concentration of imatinib that produces a 50\% reduction in tyrosine phosphorylation $\left(\mathrm{IC}_{50}\right)$ is $0.25 \mu \mathrm{M}$ for Ableson kinase (Abl) or its close family member, Abelson-related gene (Arg), and $0.3 \mu \mathrm{M}$ for the PDGF receptors (14). In contrast, no inhibition is observed for other tyrosine kinases (for example, the insulin receptor) at more than $100 \mu \mathrm{M}$. Imatinib inhibits cKit, but this is not expressed in resident LF (15). The optimal inhibitory doses of imatinib in lung and LF are known (16).

Animals. Mice bearing the PDGF-R $\alpha$-GFP construct were described (13). Production of green-fluorescent protein (GFP) is controlled by the endogenous $p d g f-r \alpha$ promoter. GFP expression in PDGF-R $\alpha$-GFP mice spatially and temporally recapitulated endogenous $p d g f-r \alpha$ expression (17). The mice used in this study carried one $p d g f-r \alpha$-GFP and one functional $p d g f-r \alpha$ allele and are phenotypically identical to WT mice, except for nuclear GFP marker (17) Protocols have been approved by the Iowa City Veterans Affairs Medical Center animal use committee (13). Aqueous imatinib, $5 \mathrm{mg} / \mathrm{kg}$, was administered by oral gavage twice daily for up to 5 successive days, ending at either P12 or P14. This dose of imatinib is optimal for specifically inhibiting PDGF-R $\alpha, \mathrm{Abl}$, or cKit in mice $(15,18)$.

Lung fibroblasts were isolated on d P12 or P14 using a modified method (13). The lung tissue was digested for $1 \mathrm{~h}$, interrupted by trituration, and the medium was replenished every $15 \mathrm{~min}$. Adherent cells were dislodged using TrypLE Express (Invitrogen, Carlsbad, CA).

Fluorescence-activated cell sorting analysis of $\alpha S M A$, Ki67, and annexin- $\boldsymbol{V}$. Freshly isolated LF were immumnostained for Ki67 and $\alpha$ SMA and subjected to fluorescence-activated cell sorting (FACS) and the 2.4G2 MAb was added to block mouse Fc receptors (13). At least 20,000 gated events were analyzed. The background fluorescence from the nonimmune $\mathrm{IgG}$ controls was subtracted. For analysis of annexin V, LF were fixed for $5 \mathrm{~min}$ at $4^{\circ} \mathrm{C}$ with $0.25 \%$ paraformaldehyde and not permeabilized. The washed LF were supplemented with AlexaFluor-647-conjugated annexin V (1:100 dilution, Invitrogen \#A23204), made $30 \mathrm{nM}$ in 4',6-diamidino-2-phenylindole (DAPI).

Tissue preparation and LSCM. Lungs were uniformly inflated, fixed, sectioned, and stained for $\alpha$ SMA (19). After blocking mouse Fc-receptors with $2.4 \mathrm{G} 2$, apoptotic cells were identified using $0.5 \mu \mathrm{g} / \mathrm{mL}$ anticleaved poly-ADP ribose polymerase (PARP, clone F21-852; BD Biosciences, Franklin Lakes, NJ) and $7.5 \mu \mathrm{g} / \mathrm{mL}$ DyLight 549 anti-mouse IgG1 (Jackson ImmunoResearch, West Grove, PA). Nuclei were stained with To-Pro3. Image $\mathrm{z}$-stacks $(3 \mu \mathrm{m}$ and $2 \mu \mathrm{m}$ intervals for $\alpha$ SMA and cleaved PARP, respectively) were acquired from randomly selected fields using a Zeiss LSM710. The laser intensities and detector gains were optimized and remained constant throughout the imaging session.

Cell culture. Mouse LF primary were isolated at P12 cultures and subcultured once (13). The medium was changed to OptiMEM-1 supplemented with $2.5 \%$ fetal bovine serum (FBS) and $\mathrm{CaCl}_{2}$ to a final calcium concentration of $3 \mathrm{mM} 24 \mathrm{~h}$ before adding $10 \mu \mathrm{M}$ imatinib, PDGF-AA $(20 \mathrm{ng} / \mathrm{mL})$, or oridonin $(2.5 \mu \mathrm{g} / \mathrm{mL})$. Imatinib and PDGF-AA were added 40 and $10 \mathrm{~min}$, respectively, before cell lysis. LF from PDGF-R $\alpha$-GFP + mice was adhered to vitronectin-coated coverslips. After $24 \mathrm{~h}$, the medium was changed to OptiMEM containing $2 \%$ FBS. Sixteen hours later, 5 ng of TGF $\beta 1$ per milliliter or imatinib was added to some coverslips, whereas others remained untreated (control). Nuclei containing GFP or Ki67 and all nuclei stained with DAPI were enumerated using fluorescence microscopy.

Analysis of ACTA2 and pdgf-r $\alpha$ mRNA using real-time quantitative $\boldsymbol{P C R}$. RNA was isolated, reverse-transcribed, and $\alpha \mathrm{SMA}, p d g f-r \alpha$ (Mm00440701_m1), and $\beta$-2-microglobulin mRNA were quantified using Taqman Gene Expression Assays (13).

Phospho Akt and PDGF-Ra immunoblotting. Washed cell layers were harvested in $10 \mathrm{mM}$ Tris-HCl, pH 7.4, $5 \mathrm{mM}$ EDTA, $50 \mathrm{mM} \mathrm{NaCl}, 50 \mathrm{mM}$ sodium fluoride, $1 \%$ Triton X-100, $1 \mathrm{mM}$ phenylmethylsulfonyl fluoride, 2 $\mathrm{mM} \mathrm{Na}_{3} \mathrm{VO}_{4}$, and $20 \mu \mathrm{g} / \mathrm{mL}$ aprotinin. Equal quantities of protein were subjected to SDS-PAGE and transferred to nitrocellulose. Rabbit polyclonal antibodies anti-phospho (Ser 473) Akt (\#9271), anti-Akt (\#9272, Cell Signal- ing Technology, Beverly, MA) or anti-phospho (Tyr 742) PDGF-R $\alpha$ (Invitrogen), and anti-PDGF-R $\alpha$ (Santa Cruz Biotechnology, Santa Cruz, CA) were diluted 1:1000 and detected using enhanced chemiluminescence.

Analyzing LSCM images. Laser scanning confocal microscopy (LSCM) z-stacks were analyzed using IPLab (BD Biosciences) (13). The pixels containing $\mathrm{Cy} 3$ dye were segmented using a red intensity range from 25 to 255 and saturation ranging from 150 to 255 . The accuracy of these ranges was confirmed by visual inspection. The composite pixel area, which contained red and met the segmentation criteria, was ascertained. The mean areas from 12 fields per lung were analyzed, using four control and four imatinib-treated mice.

The Z-stacks were analyzed using the optical fractionator and StereoInvestigator software (MicroBrightField, Williston, VT). The cells were classified as follows: nuclear GFP + (pdgf-r $\alpha$ expressing), ToPro3 + (all nuclei), cleaved PARP surrounding nuclei with ToPro3 (but without GFP), and cleaved PARP around nuclei with GFP. The approximate numbers of cells counted in the total dissector volume for each type of cell were $350,1100,2$, and 15 , respectively. Knowing the dissector volume and the lungdisplacement volume, we calculated the number of GFP + cells per lung. Six regions were analyzed, and the data were averaged for each mouse, using four mice for each age and imatinib treatment condition.

Stereology using paraffin embedded tissue. To assess the surface area of alveoli and alveolar ducts, and the arithmetic mean thickness of alveolar and alveolar duct walls, three lung slices from each mouse were embedded in paraffin, sectioned at $4.5-\mu \mathrm{m}$ intervals, and stained with hematoxylin and eosin. The surface area of the alveoli and alveolar ducts was calculated using the cycloids for $S_{\mathrm{v}}$ probe (StereoInvestigator, units, $\mu \mathrm{m}^{-1}$ ) multiplied by the displacement volume. Six fields from three separate regions of the lung (18 fields) were evaluated for each of the four control and imatinib-treated mice. The thickness of alveoli and alveolar ducts was ascertained using the point sampled intercept probe. The arithmetic mean length (thickness) was calculated as $l_{a}=\pi / 4$ (1) from randomly imaged fields (20). Nine regions from four controls and from four imatinib-treated mice were analyzed at P12 and P14 by counting 200 wall transections.

Statistical methods. Data are expressed as the mean \pm SEM of the number of different mice used or the number of different experiments performed using cell cultures. ANOVA (one-, two-, or three-way as indicated in the figure legends) was performed using Systat (Chicago, IL) and $t$ test using Microsoft Excel. Post hoc tests are described in the figure legends. Values of $p<0.05$ were considered significant.

\section{RESULTS}

Pdgfra-LF diminish after P12. Our previous studies showed that $p d g f-r \alpha$ expressing alveolar cells increased from P4 to P12 (19). To determine whether this increase was influenced by PDGF-R $\alpha$ function, we exposed mice to imatinib. Figure $1 A$ shows that imatinib diminished the abundance of GFP + alveolar cells at P12 and P14, suggesting that the maintenance of $p d g f-r \alpha$-expressing cells depends on active protein tyrosine kinase signaling (PDGF-R, Abl, or both). The abundance of $p d g f-r \alpha$-expressing cells diminished between P12 and P14 and their loss was enhanced by imatinib.

To confirm that LF comprised the GFP+ population, we isolated LF and assessed the abundance of GFP+ cells using FACS. Epithelial, macrophage, and endothelial cells comprised $8.1 \pm 6 \%(n=2), 18.1 \pm 1 \%(n=3)$, and $1.6 \pm 0.5 \%(n=3$, mean $\pm \mathrm{SD}$ ), respectively, of the cells isolated at P12 (13). Figure $1 B$ shows that at $\mathrm{P} 12$, imatinib decreased the abundance of $p d g f-r \alpha$ GFP + cells, which we previously showed were myofibroblasts (13). Exposure for $4 \mathrm{~d}$, but not 3 or fewer days was sufficient to decrease the $p d g f r \alpha$-LF at P14 (Fig. 1C).

PDGF-R $\alpha$ alters $L F$ proliferation. If PDGF-R $\alpha$-signaling is necessary to maintain LF proliferation, then the effect of imatinib should differentially alter the proliferation of $p d g f r \alpha-$ LF. We evaluated the abundance of Ki67 in freshly isolated LF using FACS. Representative FACS scatter plots for P12 are shown in Figure $2 A$, which demonstrates that the proportion of proliferative GFP + LF is lower than that of GFP- LF. 

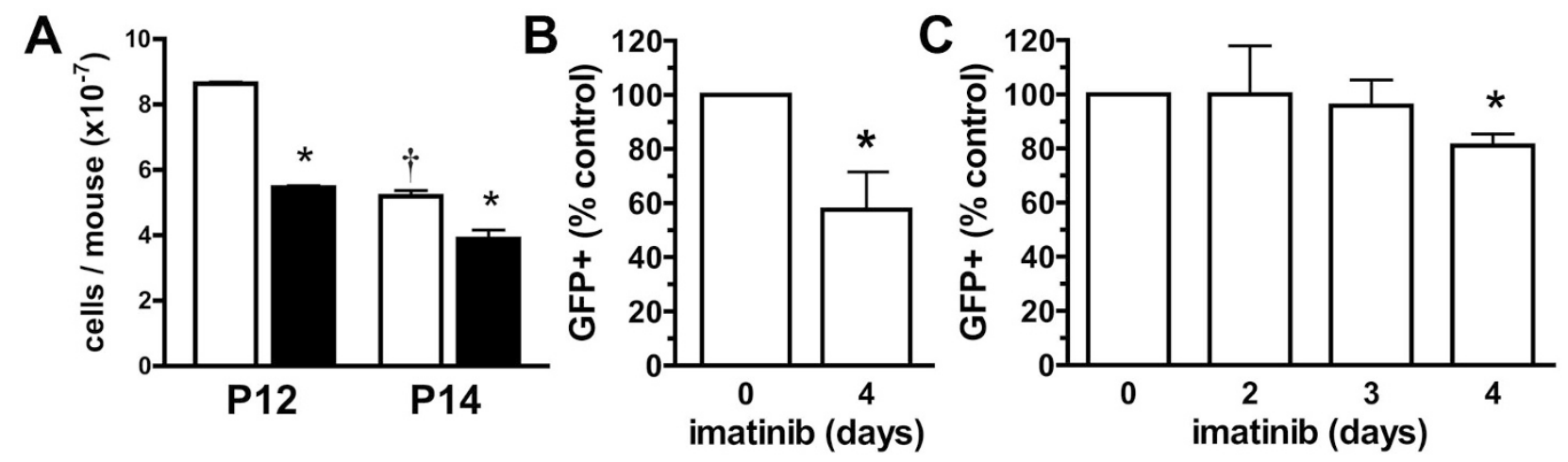

Figure 1. Imatinib decreases abundance of $p d g f r \alpha-\mathrm{GFP}+$ cells. (A) PDGF-R $\alpha(\mathrm{GFP}+)$ parenchymal cells were enumerated in LSCM z-stacks from four unexposed control $(\square)$ and four imatinib-treated (ם) lungs. Actual count $10^{7}$ fold more than shown. $* p<0.01$, imatinib-treated $v s$ age-matched control. $\dagger p<$ 0.01, P14 vs P12 control. Two-way ANOVA, Tukey test. LF isolated at P12 (B) or P14 (C) from control or imatinib-treated lungs. GFP and Ki67 containing subpopulations enumerated using FACS. $* p<0.01$, imatinib-treated $v s$ control. Two-way ANOVA, Tukey test.
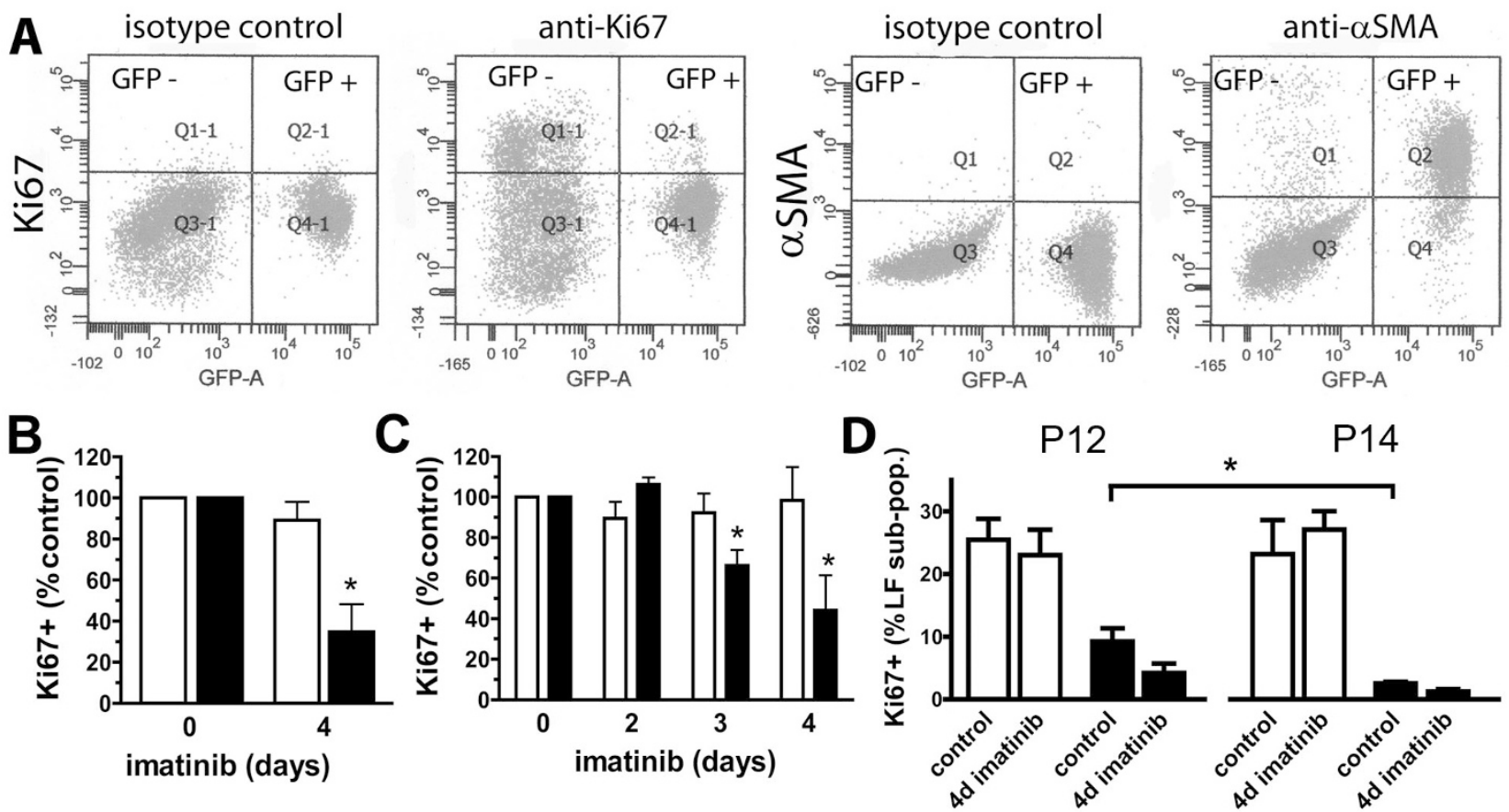

Figure 2. Imatinib reduces proliferation of $p d g f r \alpha$-LF. (A) Representative scatter plots from FACS for Ki67 and $\alpha$ SMA. $x$ axis: intensity of GFP; $y$ axis: intensities of antibody binding to Ki67 or $\alpha$ SMA. Quadrants (Q) demarcated various subpopulations. A portion of the 20,000 collected events is shown. Specifically stained GFP+, Ki67+ cells (Q2-1) ranged from 250 to 450. LF isolated from PDGF-R $\alpha$-GFP mice at P12 (B) or P14 (C) and resolved into GFP$(\square)$ and GFP $+(\square)$ by FACS. LF staining for Ki67 from mice exposed to imatinib expressed relative to concurrently stained LF from controls. ${ }^{*} p<0.01$, imatinib vs time-matched control. Two-way ANOVA, Tukey test. $(D)$ Same FACS analysis as for $(B$ and $C)$, but data expressed as percentage of cells staining with Ki67 relative to the total LF in $p d g f r \alpha$-expressing $(\mathrm{GFP}+, \mathbf{\square})$ or nonexpressing (GFP-, $\square$ ) subpopulations (subpop). $n=4$ experiments. $* p<0.05$, three-way ANOVA, Tukey test.

Nearly, all of the $\alpha$ SMA-containing LF were in the PDGF$\mathrm{R} \alpha$-GFP + subpopulation.

Figure $2 B$ and $C$ shows that exposure to imatinib suppressed proliferation in LF expressing PDGF-R $\alpha$ (GFP+, solid bars) relative to LF lacking PDGF-R $\alpha$ (GFP-) at P12 and P14, respectively. Observing that imatinib selectively suppresses proliferation in $p d g f r \alpha$-LF indicates that the effect of imatinib depends on the potential for cellular signaling through PDGF-R $\alpha$. Figure $2 D$ shows that Ki67+ cells comprise a smaller proportion of the $p d g f r \alpha$-expressing (GFP+, solid bars) than of the GFPsubpopulation. In controls, the abundance of Ki67+ LF declined from P12 to P14 only in the GFP+, but not the GFP- subpop- ulation. TGF $\beta 1$ did not increase and imatinib did not decrease proliferating Ki67+ LF, which comprised 7.5 \pm 1.4, 4.2 \pm 2.4, $5.4 \pm 0.9$, and $1.6 \pm 0.9 \%$ in control, or in cultures treated with imatinib, TGF $\beta 1$, or TGF $\beta 1$ and imatinib, respectively (mean \pm SEM, $n=3$, one-way ANOVA).

LF apoptosis is influenced by PDGF-R $\alpha$. Figure $3 A(\mathrm{P} 12)$ and $B$ (P14) shows that the proportion of apoptotic cells (bind annexin but exclude DAPI) was lower in the $p d g f-r \alpha$ expressing LF (solid bars) compared with control, suggesting that this subpopulation is more resistant to apoptosis. Exposure to imatinib made the PDGFR $\alpha$-LF (GFP+) as likely to undergo apoptosis as their nonexpressing ( $\mathrm{GFP}-$ ) counterparts. 

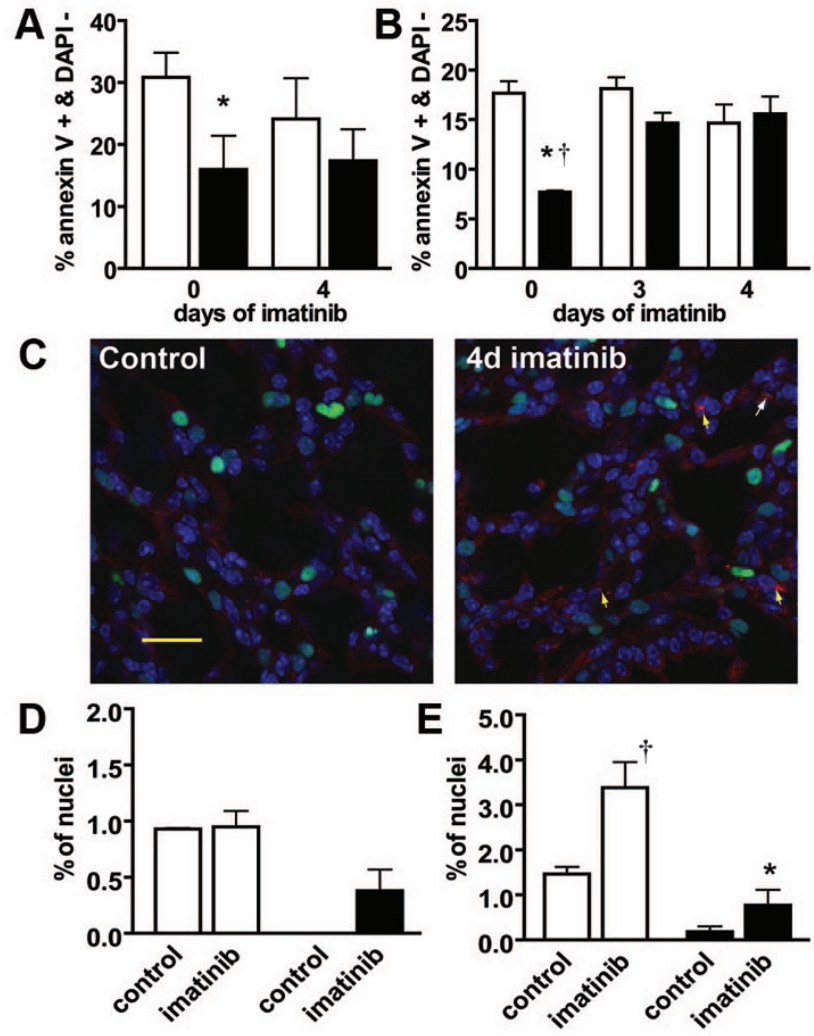

Figure 3. Functional kinase protects $p d g f r \alpha-\mathrm{LF}$ from apoptosis. Unpermeabilized-LF isolated, as in Figure 2, were subjected to FACS immediately after isolation at P12 (A) or P14 (B). Apoptotic GFP- $(\square)$ and GFP+ $(\square)$ LF stained with annexin V but not DAPI. Mean \pm SEM, $n=4$ experiments. ${ }^{*} p<0.01$, GFP + compared with time-matched GFP $-. \dagger p<0.01$, untreated $v s$ imatinib-treated GFP + LF. Two-way ANOVA, Student-Newman-Keuls test. (C) Representative micrographs (scale bar, $25 \mu \mathrm{m}$ ) from control and imatinib-treated mice stained in parallel with anticleaved PARP (red) and ToPro3 (blue to visualize all nuclei). Yellow arrows, apoptotic GFP- cells containing cleaved PARP (nuclei only visualized with ToPro3); white arrow, apoptotic PDGF-R $\alpha$ GFP + cell (green nucleus). Percentage cells containing cleaved-PARP in PDGF-R $\alpha$ expressing $(\mathrm{GFP}+, \mathbf{\square})$ compared with parenchymal cells not expressing $p d g f-r \alpha(\mathrm{GFP}-, \square)$ at P12 (D) and P14 (E). $\dagger$ Imatinib vs control GFP-; *Imatinib GFP + vs imatinib GFP $-<0.05$. One-way ANOVA, Student Newman-Keuls test.

We also assessed the abundance of cleaved PARP in lung tissue, which circumvented apoptosis resulting from the isolation procedure. Because the GFP- population also included cells that were not LF, our results partially differed from those obtained using FACS. Figure $3 C$ shows representative images obtained using LSCM with lungs from control and imatinibtreated mice at P14. Figure $3 D$ and $E$ demonstrates that apoptosis was greater in the lung parenchymal cells lacking GFP (open bars) than in the PDGF-R $\alpha$-GFP + LF at P12 and P14, respectively. As observed using FACS, apoptosis was lower in the PDGF-R $\alpha$-GFP + population compared with other parenchymal cells. However, using cleaved PARP, imatinib also increased apoptosis in alveolar cells that are not fibroblasts (and were depleted in the LF-enriched population analyzed by FACS). Inhibition of Abl promotes apoptosis in alveolar epithelial and endothelial cells $(16,21)$.

Because PDGF-R $\alpha$-signaling enhances survival through the Akt pathway, we assessed whether imatinib disrupted Akt and
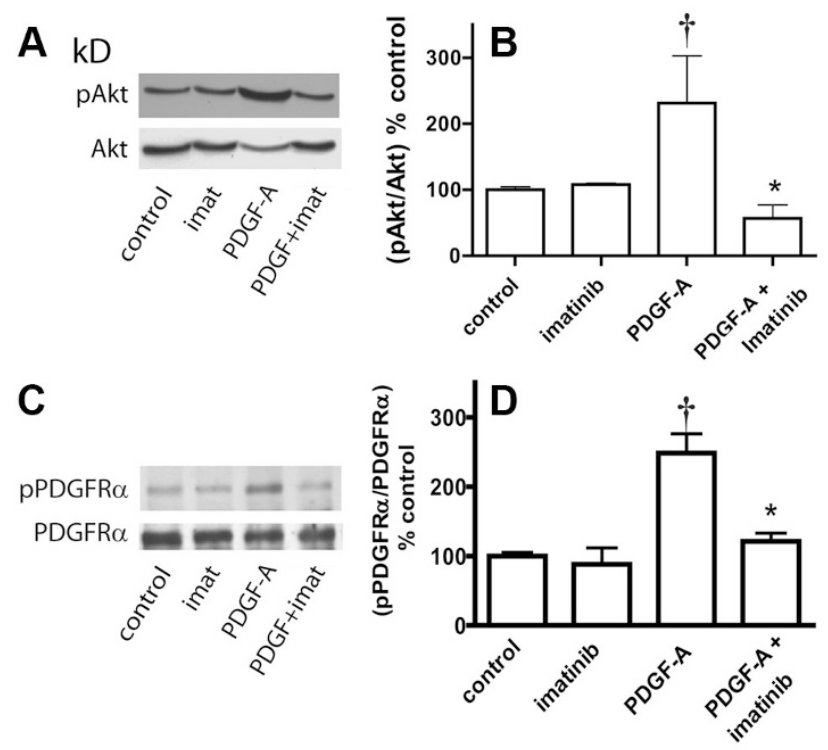

Figure 4. Imatinib reduces phospho-Akt and phospho-PDGF-R $\alpha$ in cultured mouse LF after stimulation with PDGF-A. Representative immunoblots showing phospho-Akt (pAkt) and Akt $(A)$ or phosphor-PDGF-R $\alpha$ $($ pPDGFR $\alpha)$ or PDGF-R $\alpha(C)$. Bars are means of four $(B)$ or three $(D)$ experiments. $\uparrow p<0.01$, PDGF-A $v s$ control; $* p<0.01$, PDGF-A + imatinib vs PDGF-A. Two-way ANOVA, Student-Newman-Keuls test.
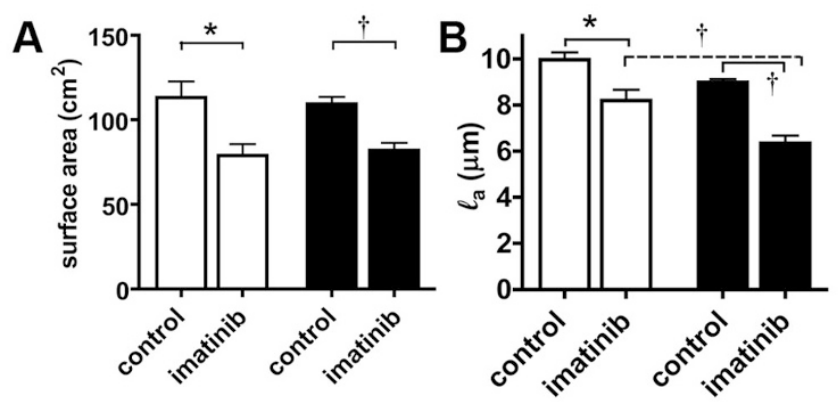

Figure 5. Imatinib reduces alveolar surface area and arithmetic mean wall

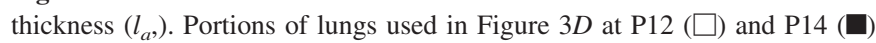
were used to analyze alveolar (alveolar and alveolar ducts were not distinguished) surface area $(A)$ wall thickness $(B)$. Four mice were used from each treatment group. $* p<0.05 ; \uparrow p<0.01$, two-way ANOVA, Tukey-test.

PDGF-R $\alpha$-phosphorylation in primary mouse LF isolated at P12 (22). Figure 4 shows that imatinib significantly reduced both Akt and PDGF-R $\alpha$ phosphorylation after PDGF-A treatment.

Imatinib reduces alveolar surface area and wall thickness. Imatinib significantly diminished both the alveolar surface area and arithmetic mean wall thickness at P12 (open bars) and P14 (solid bars; Fig. 5). Lung parenchyma comprised a similar percentage of the total lung volume in control $(90.4 \pm 0.879$, mean \pm SEM) and imatinib-exposed $(89.5 \pm 0.81)$ mice.

Nuclear factor kappa-light-chain-enhancer of activated $B$ cells $(\mathbf{N F}-\kappa \boldsymbol{B})$ regulates pdgf-r $\alpha$ transcription in $\mathbf{L F}$. The imatinib-related decrease in PDGF-R $\alpha$-GFP+ LF (Fig. 1) could result from diminished PDGF-R $\alpha$ transcription. Factors that diminish transcriptional activity of the $p d g f-r \alpha$ promoterregulatory region would also diminish the production GFP, which is directly dependent on $p d g f-r \alpha$ promoter activity. Rather than a decrease, we observed that imatinib increased 

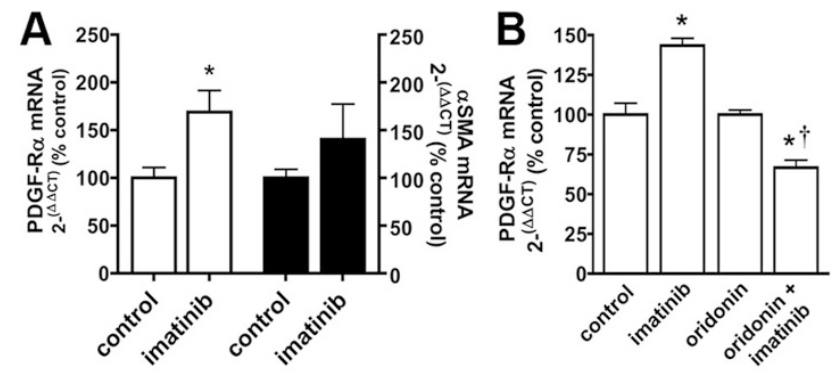

Figure 6. Imatinib increases and disruption of NF- $\kappa \mathrm{B}$-DNA binding abrogates the increase in $p d g f-r \alpha$ mRNA. (A) Mouse LF exposed to $10 \mu \mathrm{M}$ imatinib for $24 \mathrm{~h}$. RNA normalized to $\beta 2$-microglobulin mRNA and expressed relative to the control (no imatinib). $n=5$ for $p d g f-r \alpha(\square)$ and $\alpha$ SMA (ם), ${ }^{*} p<0.01$, paired $t$ test compared imatinib and control $p d g f-r \alpha$ mRNA. (B) LF exposure to imatinib and oridonin for $12 \mathrm{~h}$. * $p<0.05$, imatinib, or oridonin and imatinib $v s$ control, $\dagger p<0.05$, oridonin and imatinib $v s$ imatinib. $n=3$. Two-way ANOVA, Tukey test.

$p d g f-r \alpha$ mRNA (Fig. $6 A$ ). Oridonin disrupts binding of NF- $\kappa \mathrm{B}$ to its consensus DNA elements (23). Therefore, we reasoned that oridonin should block an imatinib-induced, NF- $\kappa \mathrm{B}$ dependent, increase in PDGF-R $\alpha$ mRNA. In the absence of oridonin, imatinib increased $p d g f-r \alpha$ mRNA, whereas in the presence of oridonin, the stimulatory effect of imatinib was overcome (Fig. 6B). Therefore, the imatinib-related decrease in GFP + alveolar cells is more likely due to a reduction in the PDGF-R $\alpha$-GFP + cell population.

Inhibition of PDGF-R and/or Abl reduces alveolar $\boldsymbol{\alpha S M A}$. Because $p d g f-r \alpha(\mathrm{GFP}+$ )-LF produce $\alpha$ SMA (Fig. $2 A$ ), we also expected that imatinib would reduce $\alpha \mathrm{SMA}$ containing LF. Figure $7 A$ shows that at P14, there is less $\alpha \mathrm{SMA}$ in alveolar ducts from a mouse treated for $5 \mathrm{~d}$ with imatinib than an untreated control. Imatinib treatment decreased the pixel area occupied by $\alpha$ SMA (Fig. 7B) (19). Using FACS (Figs. $2 A$ and $7 C$ ), we observed that the decrease in $\alpha$ SMA containing cells was only observed in the $p d g f r \alpha-\mathrm{LF}$ $(\mathrm{GFP}+)$. This indicates that PDGF-R and/or Abl/Arg signaling is required to sustain the production of $\alpha \mathrm{SMA}$. However, Figure $6 A$ shows that imatinib did not directly reduce $\alpha$ SMAgene expression in cultured LF.

\section{DISCUSSION}

PDGF-A, which signals solely through PDGF-R $\alpha$, is required for secondary alveolar septal formation and elastic fiber deposition (5). The $p d g f-r \alpha$ expressing LF population increases between P4 and P8 through proliferation (13) and maintains its abundance until at least P12 (19). We have now shown that the $p d g f r \alpha$-expressing population diminishes after P12 because of apoptosis and diminished proliferation, resulting in fewer and thinner alveolar walls. Protein tyrosine kinase signaling through PDGF-R and/or Abl/Arg is important for sustaining the $p d g f r \alpha$-LF subpopulation (Fig. 8). Because imatinib increased, rather than decreased $p d g f r \alpha$ gene expression in vitro, it is more likely that the loss of $p d g f r \alpha$-expressing cells results from a decline in the number of cells rather than a decrease in $p d g f-r \alpha$ transcription. PDGF-A stimulates and imatinib interferes with the ability of PDGF-A to increase PDGF-R $\alpha$ - and Akt-
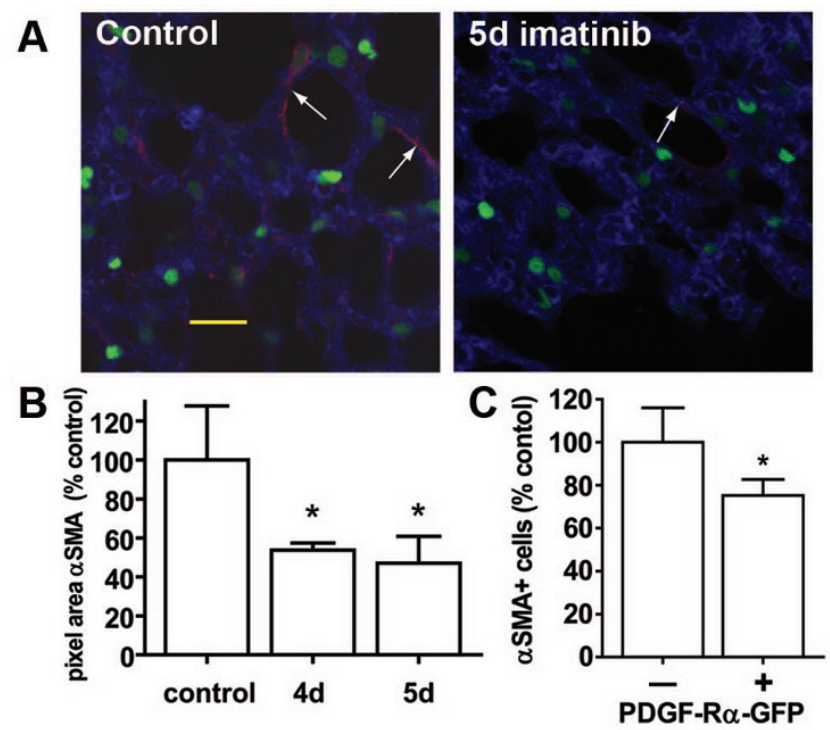

Figure 7. Imatinib reduces alveolar $\alpha$ SMA. (A) Representative images of control and imatinib-treated mice. Lung tissues stained in parallel with Cy3-conjugated anti- $\alpha$ SMA (red). AlxaFluor 633 conjugated DiD (blue) counterstain. White arrows, $\alpha$ SMA; scale bar, $25 \mu \mathrm{m}$. (B) Summary $\alpha$ SMA pixel areas using three mice per treatment group. ${ }^{*} p<0.01$, imatinib-treated ( 4 or 5 d) $v s$ control. $(C)$ Comparison of $\alpha$ SMA (using FACS) of untreated control and imatinib-treated within the PDGF-R $\alpha$ negative $(-, \mathrm{GFP}-)$ or within the PDGF-R $\alpha$-positive $(+, \mathrm{GFP}+)$ subpopulations. Same LF preparations as for P14 in Figure $2 C$, stained for $\alpha$ SMA. ${ }^{*} p<0.05$, two-way ANOVA, Student Newman-Keuls test.

\section{septal thinning \& MF apoptosis PDGF-A / PDGF-R $\alpha$}

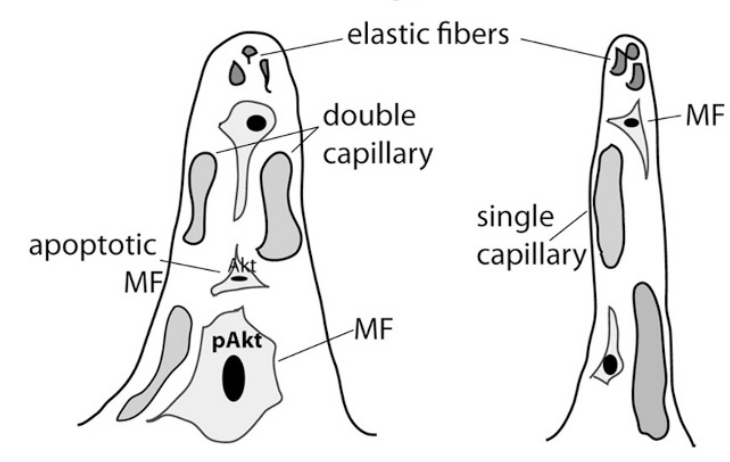

Figure 8. Alveolar myofibroblasts $(\mathrm{MF})$ diminish during secondary septal maturation. From P12 to P14 (proceeding from left to right), signaling through PDGF-R $\alpha$ diminishes (shaded triangle). Fewer $p d g f-r \alpha$ expressing MF (black nuclei) occupy the central core because attenuation of PDGF-R $\alpha$ signaling results in decreased proliferation and increased apoptosis (less phospho-Akt, pAkt). Septal thinning enables the capillary network to coalesce and closely appose the epithelial septal covering.

phosphorylation, suggesting that the antiapoptotic effect of PDGF-A on $p d g f r \alpha$-LF may involve Akt.

Others demonstrated that without PDGF-A, $p d g f-r \alpha$ expressing LF diminish and secondary septal formation fails (5). However, these studies did not establish whether the effects of PDGF-A were specifically directed toward $p d g f r \alpha-\mathrm{LF}$ or toward progenitors, which supplied the $p d g f r \alpha$-LF population. Our novel findings indicate that protein tyrosine kinase sig- 
naling through PDGF-R and/or Abl maintains the $p d g f r \alpha$ myofibroblast subpopulation and that diminished PDGF-R $\alpha$ signaling may promote septal thinning and maturation of the air-blood interface. This diminution is accelerated by imatinib.

Using imatinib to disrupt protein tyrosine kinase signaling has distinct advantages and some disadvantages. Targeted gene deletions of PDGF-A or PDGF-R $\alpha$ are gene and pathway specific, but they do not disrupt a particular phase of alveolar development, because the abnormalities begin in utero. Therefore, the observations made postnatally are compounded by abnormalities before P4, when septal eruption begins. Starting imatinib on or after P8 enabled septal eruption and early elongation to proceed normally, so we could specifically study subsequent septal elongation and thinning. A disadvantage is that although imatinib is selective, it likely inhibits PDGF-R $\beta$ and $\mathrm{Abl}$, as well as PDGF-R $\alpha$, under our experimental conditions.

Nevertheless, inhibition of PDGF-R $\alpha$ most likely accounts for our observations. Imatinib inhibited proliferation and enhanced apoptosis in the PDGF-R $\alpha$-GFP+ but not the GFPsub-population, indicating that PDGF-R $\alpha$-kinase is necessary for the observed effects. Whether inhibition of PDGF-R $\alpha$ is sufficient to account for our findings is less certain, because imatinib also inhibits Abl kinase. In fibroblasts, PDGFreceptors primarily activate Abl-kinase by signaling through Src and phospholipaseC- $\gamma$, rather than through phosphoinositol-3-kinase, the dominant PDGF-R $\alpha$-pathway (24). This suggests that PDGF-R $\alpha$ may not initiate Abl-mediated signaling in LF. Although Abl is present in cultured mouse LF, its constitutive kinase activity is very low, and we could not demonstrate further reduction by imatinib (data not shown). Although others observed that inhibition of Abl by imatinib reduced TGF $\beta$-mediated renal fibroblast proliferation (25), we have not observed this in mouse LF. We previously showed that proliferation decreased in $p d g f r \alpha$-expressing LF at P12, whereas Smad-dependent signaling did not (13). Therefore, it is unlikely that imatinib interferes with activation of Ablkinase by TGF $\beta$, in our mouse LF cultures. Because PDGF-A enhances Akt-phosphorylation, $p d g f r \alpha$-LF differentially maintains survival as long as signaling through PDGF-R $\alpha$ is maintained. But as proliferation continues to decline, $p d g f r \alpha-\mathrm{LF}$ diminishes so that septal thinning occurs (Fig. 8).

In conclusion, we have shown that $p d g f-r \alpha$ expressing myofibroblasts persist throughout the period of maximal septation (P12) and then diminish. Although $p d g f r \alpha$-GFP expressing myofibroblasts are less proliferative than their GFP- counterparts at P12, they are more resistant to apoptosis. Timely regulation of cellular proliferation and apoptosis is required to ensure that sufficient myofibroblasts are present not only to establish mechanically competent septa but also to enable septal thinning as the vasculature matures. Aberrant regulation occurs during positive pressure mechanical ventilation of newborn mammals and likely contributes to the development of BPD (12). Targeted and carefully timed strategies to maintain a normal complement of myofibroblasts could improve the outcome for children with BPD. Similar strategies may be relevant to pulmonary emphysema in adults, when the alveolar septa atrophy through apoptosis (26). Alternatively, insufficient myofibroblast apoptosis contrib- utes to pulmonary fibrosis and may be corrected by the administration of imatinib (27).

\section{REFERENCES}

1. Mund SI, Stampanoni M, Schittny JC 2008 Developmental alveolarization of the mouse lung. Dev Dyn 237:2108-2116

2. Nicola T, Hagood JS, James ML, MacEwen MW, Williams TA, Hewitt MM, Schwiebert L, Bulger A, Oparil S, Chen YF, Ambalavanan N 2009 Loss of Thy-1 inhibits alveolar development in the newborn mouse lung. Am J Physiol Lung Cell Mol Physiol 296:L738-L750

3. Srinivasan S, Strange J, Awonusonu F, Bruce MC 2002 Insulin-like growth factor I receptor is downregulated after alveolarization in an apoptotic fibroblast subset. Am J Physiol Lung Cell Mol Physiol 282:L457-L467

4. Mitchell JJ, Reynolds SE, Leslie KO, Low RB, Woodcock-Mitchell J 1990 Smooth muscle cell markers in developing rat lung. Am J Respir Cell Mol Biol 3:515-523

5. Boström H, Willetts K, Pekny M, Levéen P, Lindahl P, Hedstrand H, Pekna M, Hellström M, Gebre-Medin S, Schalling M, Nilsson M, Kurland S, Törnell J, Heath JK, Betsholtz C 1996 PGDF-A signaling is a critical event in lung alveolar myofibroblast development and alveogenesis. Cell 85:863-873

6. Buch S, Han RN, Cabacungan J, Wang J, Yuan S, Belcastro R, Deimling J, Jankov R, Luo X, Lye SJ, Post M, Tanswell AK 2000 Changes in expression of plateletderived growth factor and its receptors in the lungs of newborn rats exposed to air or $60 \% \mathrm{O}(2)$. Pediatr Res 48:423-433

7. Olson LE, Soriano P 2009 Increased PDGFR $\alpha$ activation disrupts connective tissue development and drives systemic fibrosis. Dev Cell 16:303-313

8. Li J, Hoyle GW 2001 Overexpression of PDGF-A in the lung epithelium of transgenic mice produces a lethal phenotype associated with hyperplasia of mesenchymal cells. Dev Biol 239:338-349

9. Burri PH 2006 Structural aspects of postnatal lung development-alveolar formation and growth. Biol Neonate 89:313-322

10. Wu S, Platteau A, Chen S, McNamara G, Whitsett J, Bancalari E 2010 Conditional overexpression of connective tissue growth factor disrupts postnatal lung development. Am J Respir Cell Mol Biol 42:552-563

11. Thibeault DW, Mabry SM, Ekekezie II, Zhang X, Truog WE 2003 Collagen scaffolding during development and its deformation with chronic lung disease. Pediatrics 111:766-776

12. Coalson JJ 2006 Pathology of bronchopulmonary dysplasia. Semin Perinatol 30:179-184

13. Kimani PW, Holmes AJ, Grossmann RE, McGowan SE 2009 PDGF-R $\alpha$ gene expression predicts proliferation, but PDGF-A suppresses transdifferentiation of neonatal mouse lung myofibroblasts. Respir Res 10:119-135

14. Druker BJ, Tamura S, Buchdunger E, Ohno S, Segal GM, Fanning S, Zimmermann J, Lydon NB 1996 Effects of a selective inhibitor of the Abl tyrosine kinase on the growth of Bcr-Abl positive cells. Nat Med 2:561-566

15. Vuorinen K, Gao F, Oury T, Kinnula VL, Myllärniemi M 2007 Imatinib mesylate inhibits fibrogenesis in asbestos-induced interstitial pneumonia. Exp Lung Res 33:357-373

16. Vittal R, Zhang H, Han MK, Moore BB, Horowitz JC, Thannickal VJ 2007 Effects of the protein kinase inhibitor, imatinib mesylate, on epithelial/mesenchymal phenotypes: implications for treatment of fibrotic diseases. J Pharmacol Exp Ther 321:35-44

17. Hamilton TG, Klinghoffer RA, Corrin PD, Soriano P 2003 Evolutionary divergence of platelet derived growth factor alpha receptor signaling mechanisms. Mol Cell Biol 23:4013-4025

18. Krebs R, Tikkanen JM, Nykänen AI, Wood J, Jeltsch M, Yla-Herttuala S, Koskinen PK, Lemström KB 2005 Dual role of vascular endothelial growth factor in experimental obliterative bronchiolitis. Am J Respir Crit Care Med 171:1421-1429

19. McGowan SE, Grossmann RE, Kimani PW, Holmes AJ 2008 Platelet-derived growth factor receptor-alpha-expressing cells localize to the alveolar entry ring and have characteristics of myofibroblasts during pulmonary alveolar septal formation. Anat Rec (Hoboken) 291:1649-1661

20. Weibel ER 1979-1980 Stereological Methods. Academic Press, London, pp 9-196

21. Evensen L, Micklem DR, Blois A, Berge SV, Aarsaether N, Littlewood-Evans A, Wood J, Lorens JB 2009 Mural cell associated VEGF is required for organotypic vessel formation. PLoS One 4:e5798

22. Horowitz JC, Rogers DS, Sharma V, Vittal R, White ES, Cui Z, Thannickal VJ 2007 Combinatorial activation of FAK and AKT by transforming growth factor- $\beta 1$ confers an anoikis-resistant phenotype to myofibroblasts. Cell Signal 19:761-771

23. Zhang N, Khachigian LM 2009 Injury-induced platelet-derived growth factor receptor-alpha expression mediated by interleukin-1beta (IL-1beta) release and cooperative transactivation by NF-kappaB and ATF-4: IL-1beta facilitates HDAC-1/2 dissociation from promoter. J Biol Chem 284:27933-27943

24. Plattner R, Pendergast AM 2003 Activation and signaling of the Abl tyrosine kinase: bidirectional link with phosphoinositide signaling. Cell Cycle 2:273-274

25. Wilkes MC, Leof EB 2006 Transforming growth factor $\beta$ activation of $\mathrm{c}-\mathrm{Abl}$ is independent of receptor internalization and regulated by phosphatidylinositol 3-kinase and PAK2 in mesenchymal cultures. J Biol Chem 281:27846-27854

26. Aoshiba K, Nagai A 2009 Senescence hypothesis for the pathogenic mechanism of chronic obstructive pulmonary disease. Proc Am Thorac Soc 6:596-601

27. Garneau-Tsodikova S, Thannickal VJ 2008 Protein kinase inhibitors in the treatment of pulmonary fibrosis. Curr Med Chem 15:2632-2640 